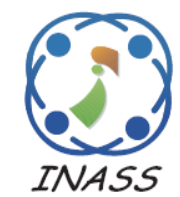

\title{
Application of Several Artificial Intelligence Models for Forecasting Meteorological Drought Using the Standardized Precipitation Index in the Saïss Plain (Northern Morocco)
}

\author{
Abdelhamid El Ibrahimi ${ }^{*}$ \\ Abdennasser Baali $^{1}$ \\ ${ }^{1}$ Faculty of Sciences dher el mahrez, Department of geology, Laboratory of Geosystems, \\ Environment and Sustainable Development, Sidi Mohamed Ben Abdellah University, \\ $\mathrm{Fez}, \mathrm{Morocco}$ \\ * Corresponding author’s Email: abdelhamidsvt@gmail.com
}

\begin{abstract}
Drought is one of the main natural factors influencing different aspects of human life. Over the decades, intelligent techniques have proven to be very capable of modeling and predicting nonlinear and dynamic time series. Therefore, the present study aims to predict drought by using and comparing neuro-fuzzy adaptive inference systems (ANFIS), artificial neural network of multilayered perceptron (ANN-MLP) and the support vector model (SVR). For this purpose, the precipitation data obtained from the Ain Bittit station were used for a statistical period of 34 years. In addition, the short-term ( 3 and 6 months) and long-term (9 and 12 months) time scales were calculated using the standardized precipitation index (SPI). Then, depending on the results of the calculations, the period 1979-2000 was selected as a control group and the period 2003-2012 was selected as an experimental group. In order to predict the SPI for the $(t+1)$ period, SPI values, precipitation from previous months were used. The results indicated that, in the majority of time scales, the ANFIS model gives SPI values and predictive dryness more accurately than the SVR, and ANN models.
\end{abstract}

Keywords: Drought, ANFIS, SPI, ANN-MLP, SVR.

\section{Introduction}

The main indication of meteorological drought is the decline in precipitation to below normal precipitation (long-term average precipitation).

Various indices were presented to determine the characteristics of meteorological drought. In this research, the standardized precipitation index (SPI) was used as one of the most important and important indices available. Previous studies on this concept were as follows: [1] used the adaptive neuro-fuzzyinference system (ANFIS) to predict drought in Turkey. Several SPI prediction models were examined in 1, 3, 6, 9 and 12 months. Examination results show that a combination of precipitation and SPI improves the performance of the models. Comparisons of the results of the linear regression (FFNN) and multiple linear regression (MLR) methods revealed the superiority of ANFIS on the other two models. [2] Also used ANFIS and fuzzy logic models to predict meteorological drought. They used SPI to analyze drought and also used 3, 6, 9, and 12 months SPIs to better predict drought. The results of their research also reflected the superiority of ANFIS on the model of fuzzy logic. [3] Used a fuzzy inference system and neural networks perceptron multilayers to predict the level of groundwater resources in Kerman Plain. The results of this research have proved the superiority of ANFIS on the perceptible network of multilayers.

While modern remote sensing, spatial modeling and geographic information technologies help users detect, simulate and predict environmental change, this technology is not yet well integrated with multilevel cooperative responses. New techniques such as Artificial Neural Networks (ANN), Fuzzy Logic 


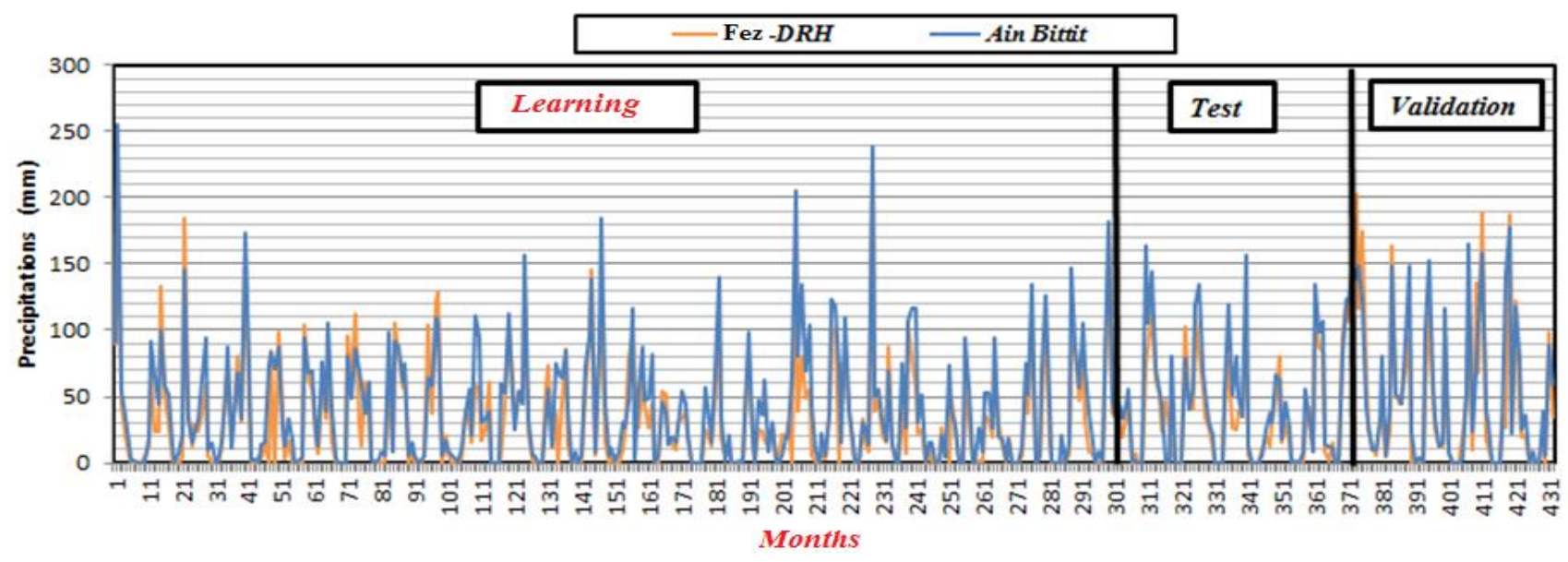

Figure.1 Time series of monthly precipitation data for the two stations of Fez-DRH and Ain Bittit for the period 1978/79 to $2013 / 14$

(FL) and ANFIS have recently been accepted as an effective modeling tool for complex hydrological systems and widely used for prediction.

In addition, [4] used the ANFIS, ANN, WaveletANN and Wavelet-ANFIS models to forecast weather drought over the next 3 months on the basis of SPI for the province of Azerbaijan (Iran). The results of this research indicated that all the modeling methods considered could have SPI, but the Wavelet-ANFIS hybrid model showed better performance. The multi-layered perceptron neural network (ANN-MLP) has been studied in five Iranian synoptic stations. The higher quantitative values of SPI using the Levenberg-Marquardt optimized algorithm (LMA) and the tangent of the asmogic function. The studies were able to calculate the SPI3, SPI6, SPI9, SPI12 and SPI24 values. In these studies, the ANN-MLP of the 1-month prediction SPIs was also examined. The results showed that the ANN-MLP model was capable of predicting 12- and 24-month SPIs with greater accuracy than other SPIs [5]. [6] also worked on population prediction based on Artificial Neural Networks (RNAs) of three regions in the San Francisco River basin, Brazil. The results of their research showed that the method is capable of predicting SPI for the coming month within 12 months, but it loses [7] used satellite imagery, climate indices and artificial neural networks to predict drought. [8] Calculated the SPIs of the Mamloo and Taleqan watersheds in Tehran, Iran. Depending on the meteorological variables, including temperature and geo-potential height, they found that SVM often gives very accurate results. Therefore, this method can be used to predict the non-linear behavior of meteorological data in shortterm statistical periods. In a research, [9] studied the potential of the support vector machine to predict the level of water contained in Lake Erie in the longrun (12 months). They also made comparisons between SVM, ANN-MLP and an autoregressive model. The results of their studies suggested that SVM showed better performance than the other two models.

The objective of this study was to develop an appropriate model for preventing meteorological drought using the Standardized Forecast Index. To this end, the time series data for the period 1979 to 2014 were used in this research.

\section{Methods and materials}

The objective of this study was to develop an appropriate predictive model of meteorological drought using the Standardized Precipitation Index. To this end, data from the time series between 1978/79 and 2013/14, recorded on a monthly basis, of the two Ain Bittit and Fez-DRH stations were used in this research.

\subsection{Data}

In this study, SPI values and precipitation from previous months are used to generate a drought estimation model using the ANFIS method. For this, the SPI outputs for 1, 3, 6, 9 and 12 months were considered.

\subsection{Methods}

\subsubsection{Models ANN-MLP}

The ANN-MLP models for drought prediction were developed using the MATLAB R2010 software. In this paper, two networks have been constructed including the feed-forward neural network (FNN) and Elman or the recurrent neural network (RNN). 
Table 1. Training parameters for ANFIS models

\begin{tabular}{cc}
\hline AND method & Prod \\
\hline Imp. method & Minimum \\
Aggr. method & Maximum \\
Defuzzification method & Wtaver \\
Step size & 0,21 \\
Rate of decrease in step size & 0,8 \\
Step size increase rate & 1,20 \\
Membership function & General bell belonging function $(\mathrm{m}=3)$ \\
\hline
\end{tabular}

For each stage of optimization, if performance decreases, the learning rate increases. This is probably the simplest and most common way to form a network [10]. Similarly, the LM method is a modification of Newton's classical algorithm to find an optimal solution to a minimization problem. The BR is an algorithm that automatically sets the optimal values for the objective function parameters. The advantage of this algorithm is that whatever the size of the network, the function will not be overadjusted [11]. The current SPI data was imported as input and the SPI for one, two and three times (12 months) to come was considered a target.

\subsubsection{Models SVR}

SVR models adhere to the principle of minimization of structural risk as opposed to the empirical risk minimization principle used by classical neural networks [12]. Therefore, these models reduce the generalization error as opposed to the learning error. The development of SVR models can be found in [13]. All SVR models were created using the Online SVR software created by [14], which can be used to build vector support machines for regression.

The data was divided into two sets: a calibration set and a validation set. $90 \%$ of the data was allocated to the calibration set while the final $10 \%$ of the data was used as a validation set. RBF, polynomial and linear functions were applied as kernel functions for the prediction of SVR precipitation in this study. As a consequence, each SVR model consisted of three selected parameters: gamma $(\gamma)$, cost $(\mathrm{C})$ and $\varepsilon s p(\varepsilon)$. The parameter $\gamma$ is a constant that reduces the space of the model and controls the complexity of the solution, while (C) is a positive constant which is a capacity control parameter, and $\varepsilon$ is the loss function that describes the vector of regression without all input data [15]. These three parameters were selected on the basis of a test and error procedure.

\subsubsection{Models ANFIS}

The ANFIS parameters are adjusted during the learning phase. To do this, a data set associating the input and output sequences is required. For the implementation of this phase, the hybrid learning algorithm is used. The learning is done in an iterative manner until the number of training cycles is reached or until the average error between the desired output value and generated by the ANFIS reaches a predetermined value. This phase therefore depends on the quality of the set of data in the sense that this set must best represent the different expected behaviors ([16]).

The ANFIS model eliminates the "black box" effect of classical neural networks, associates dysfunctional knowledge available in the form of fuzzy rules and preserves a learning capacity derived from neural networks. One of the most important steps for the generation of neural-fuzzy network structure (ANFIS) is the establishment of fuzzy inference rules. Four different membership functions (MF) have been tested for the ANFIS models in this work, ie Gaussian (MFgauss), bellshaped (MFgbell), triangular (MFtri), spline-based (MFpi) or Piduetoits [17]. ANFIS models with different types of MF were run with 2, 3, 4 and 5 MF and with 50, 100, 150, 200, 250, 300 and 400 iterations for each input data node [18]. The characteristics of the ANFIS model formation are presented in table 1 . The current SPI data were imported as inputs and the ITS for 1, 2 and 3 times (12 months) to come were considered as a target.

\section{Main title}

\subsection{Series forecasts SPI-3 and SPI-12}

The statistics on the predictive performance of all models in the learning and test phases are compared by RMSE and R2 in tables 2, 3, 4 and 5. In general, it is found that all models produce sufficient predictions, particularly at scales 1 and 2- 
Table 2. The results of the ANN-MLP, SVR and ANFIS models for SPI-3 prediction for 1, 2 and 3-months

\begin{tabular}{|c|c|c|c|c|c|c|c|c|}
\hline \multicolumn{9}{|c|}{ SPI-3 } \\
\hline \multicolumn{9}{|c|}{ 1-month } \\
\hline Station & \multicolumn{4}{|c|}{ Fez-DRH } & \multicolumn{4}{|c|}{ Ain Bittit } \\
\hline \multirow{2}{*}{ Methods } & \multicolumn{2}{|c|}{ Learning } & \multicolumn{2}{|c|}{ Test } & \multicolumn{2}{|c|}{ Learning } & \multicolumn{2}{|c|}{ Test } \\
\hline & RMSE & $\mathrm{R}^{2}$ & RMSE & $\mathrm{R}^{2}$ & RMSE & $\mathrm{R}^{2}$ & RMSE & $\mathrm{R}^{2}$ \\
\hline SVR & 0.546 & 0.7253 & 0.406 & 0.7628 & 0.402 & 0.7747 & 0.508 & 0.773 \\
\hline ANN-MLP & 0.515 & 0.7742 & 0.404 & 0.7506 & 0.423 & 0.7022 & 0.509 & 0.762 \\
\hline ANFIS & 0.242 & 0.7902 & 0.139 & 0.7825 & 0.170 & 0.7785 & 0.309 & 0.798 \\
\hline \multicolumn{9}{|c|}{ 2-month } \\
\hline Station & \multicolumn{4}{|c|}{ Fez-DRH } & \multicolumn{4}{|c|}{ Ain Bittit } \\
\hline \multirow{2}{*}{ Méthode } & \multicolumn{2}{|c|}{ Learning } & \multicolumn{2}{|c|}{ Test } & \multicolumn{2}{|c|}{ Learning } & \multicolumn{2}{|c|}{ Test } \\
\hline & RMSE & $\mathrm{R}^{2}$ & RMSE & $\mathrm{R}^{2}$ & RMSE & $\mathrm{R}^{2}$ & RMSE & $\mathrm{R}^{2}$ \\
\hline SVR & 0.675 & 0.690 & 0.582 & 0.689 & 0.574 & 0.685 & 0.574 & 0.688 \\
\hline ANN-MLP & 0.602 & 0.624 & 0.510 & 0.610 & 0.598 & 0.620 & 0.515 & 0.631 \\
\hline ANFIS & 0.329 & 0.748 & 0.236 & 0.719 & 0.212 & 0.739 & 0.234 & 0.761 \\
\hline \multicolumn{9}{|c|}{ 3-month } \\
\hline Station & \multicolumn{4}{|c|}{ Fez-DRH } & \multicolumn{4}{|c|}{ Ain Bittit } \\
\hline \multirow{2}{*}{ Methods } & \multicolumn{2}{|c|}{ Learning } & \multicolumn{2}{|c|}{ Test } & \multicolumn{2}{|c|}{ Learning } & \multicolumn{2}{|c|}{ Test } \\
\hline & RMSE & $\mathrm{R}^{2}$ & RMSE & $\mathrm{R}^{2}$ & RMSE & $\mathrm{R}^{2}$ & RMSE & $\mathrm{R}^{2}$ \\
\hline SVR & 0.817 & 0.577 & 0.718 & 0.574 & 0.744 & 0.525 & 0.740 & 0.548 \\
\hline ANN-MLP & 0.829 & 0.504 & 0.739 & 0.514 & 0.703 & 0.512 & 0.735 & 0.524 \\
\hline ANFIS & 0.531 & 0.620 & 0.341 & 0.606 & 0.305 & 0.617 & 0.337 & 0.721 \\
\hline
\end{tabular}

months in advance and low on a 3-month scale in both the learning and testing phases. Before SPI series pre-processing, the tables $2,3,4$ and 5 illustrate the best results of the SVR, ANN and ANFIS models in terms of learning and testing for the SP-3 and SP-12 time series. The tables show that the ANFIS model presents better SVR and ANN predictions with smaller RMSE values and higher R2 values than SVR and ANN models, particularly for 1 and 2 months in advance. On the other hand, the forecast accuracy of the SVR and ANN models is very close to all the forecasting times, although the ANN method has a slight superiority compared to the SVR. It is found that all four models demonstrated acceptable performance, whereas the ANFIS model indicates a good forecast over the 3 time periods at the two station levels. These results show that the ANFIS model more easily captures the characteristics and trends of the model input changes. However, the best SPI-3s are indicated for a period of 1 month.

The analysis of the coefficient of determination R2 and RMSE for both the learning and test phase (tables 2, 3, 4 and 5) indicates a decrease in performance from learning to testing. Although, the performance of different models decreases as the forecast time increases.

However, as shown in tables 2 and 3, the use of wavelet transforms improved the predictive capacity of the models, justified by lower RMSE values for the WA-SVR WA-ANN and WA-ANFIS models, to the SVR, ANN and ANFIS models. The RMSE ranged from 0.546 to 0.163 , from 0.515 to 0.144 and from 0.242 to 0.130 in apprenticeship, from 0.406 to 0.195 , from 0.404 to 0.156 and from 0.139 to 0.125 in the test phase at the 1 -month period in advance for WA-SVR, WA-ANN and WA-ANFIS respectively. Although the majority of SPI-3 forecasts have lower values in terms of R2 and higher in terms of RMSE than SPI-12, when the forecast period is extended to 3 months, predictions deteriorate predominantly in the majority of the predictive measurements for the two stations in the learning and testing phase. A possible explanation for the low correlation between predicted and observed SPI-3 values is the low level of autocorrelation between the data set, while there is better autocorrelation in the SPI-12 time series when the time increases. For SPI 3 and other short-term SPIs, each new month has a significant impact on the sum of precipitation. Therefore, SPI 3 is sensitive to any change in precipitation from one month to another, which is a possible explanation for why SPI 3 prediction results have lower R2 values. In the case of SPI 12, each month has no impact on the total and the index is not as sensitive to changes in precipitation from one month to another. The fact that SPI 3 is more sensitive to changes in precipitation yields less accurate forecasting results than SPI-12. However, the effects of wavelet analysis are more significant for SPI-3 than for SPI-12, especially for 1-month forecast times. 
Table 4. Best results for the 1, 2 and 3-month models of ANN-MLP, SVR and ANFIS for SPI-12 prediction

\begin{tabular}{|c|c|c|c|c|c|c|c|c|}
\hline \multicolumn{9}{|c|}{ SPI-12 } \\
\hline \multicolumn{9}{|c|}{ 1-month } \\
\hline Station & \multicolumn{4}{|c|}{ Fez-DRH } & \multicolumn{4}{|c|}{ Ain Bittit } \\
\hline \multirow{2}{*}{ Methods } & \multicolumn{2}{|c|}{ Learning } & \multicolumn{2}{|c|}{ Test } & \multicolumn{2}{|c|}{ Learning } & \multicolumn{2}{|c|}{ Test } \\
\hline & RMSE & $\mathrm{R}^{2}$ & RMSE & $\mathrm{R}^{2}$ & RMSE & $\mathrm{R}^{2}$ & RMSE & $\mathrm{R}^{2}$ \\
\hline SVR & 0.432 & 0.789 & 0.311 & 0.7921 & 0.307 & 0.7987 & 0.413 & 0.7911 \\
\hline ANN-MLP & 0.445 & 0.740 & 0.309 & 0.8056 & 0.328 & 0.7152 & 0.414 & 0.8003 \\
\hline ANFIS & 0.301 & 0.815 & 0.115 & 0.8725 & 0.105 & 0.8425 & 0.081 & 0.8550 \\
\hline \multicolumn{9}{|c|}{ 2-month } \\
\hline Station & \multicolumn{4}{|c|}{ Fez-DRH } & \multicolumn{4}{|c|}{ Ain Bittit } \\
\hline \multirow{2}{*}{ Methods } & \multicolumn{2}{|c|}{ Learning } & \multicolumn{2}{|c|}{ Test } & \multicolumn{2}{|c|}{ Learning } & \multicolumn{2}{|c|}{ Test } \\
\hline & RMSE & $\mathrm{R}^{2}$ & RMSE & $\mathrm{R}^{2}$ & RMSE & $\mathrm{R}^{2}$ & RMSE & $\mathrm{R}^{2}$ \\
\hline SVR & 0.577 & 0.783 & 0.493 & 0.794 & 0.479 & 0.790 & 0.476 & 0.793 \\
\hline ANN-MLP & 0.504 & 0.727 & 0.415 & 0.715 & 0.499 & 0.725 & 0.417 & 0.735 \\
\hline ANFIS & 0.331 & 0.791 & 0.141 & 0.823 & 0.183 & 0.844 & 0.136 & 0.8440 \\
\hline \multicolumn{9}{|c|}{ 3-month } \\
\hline Station & \multicolumn{4}{|c|}{ Fez-DRH } & \multicolumn{4}{|c|}{ Ain Bittit } \\
\hline \multirow{2}{*}{ Methods } & \multicolumn{2}{|c|}{ Learning } & \multicolumn{2}{|c|}{ Test } & \multicolumn{2}{|c|}{ Learning } & \multicolumn{2}{|c|}{ Test } \\
\hline & RMSE & $\mathrm{R}^{2}$ & RMSE & $\mathrm{R}^{2}$ & RMSE & $\mathrm{R}^{2}$ & RMSE & $\mathrm{R}^{2}$ \\
\hline SVR & 0.719 & 0.680 & 0.623 & 0.679 & 0.649 & 0.630 & 0.642 & 0.651 \\
\hline ANN-MLP & 0.731 & 0.607 & 0.644 & 0.619 & 0.608 & 0.619 & 0.637 & 0.627 \\
\hline ANFIS & 0.433 & 0.723 & 0.246 & 0.783 & 0.255 & 0.757 & 0.233 & 0.791 \\
\hline
\end{tabular}

Table 5. The results of the SPI-12 prediction models WA-ANN-MLP, WA-SVR and WA-ANFIS for 1, 2 and 3 months

\begin{tabular}{|c|c|c|c|c|c|c|c|c|}
\hline \multicolumn{9}{|c|}{ SPI-12 } \\
\hline \multicolumn{9}{|c|}{ 1-month } \\
\hline Station & \multicolumn{4}{|c|}{ Fez-DRH } & \multicolumn{4}{|c|}{ Ain Bittit } \\
\hline \multirow{2}{*}{ Methods } & \multicolumn{2}{|c|}{ Learning } & \multicolumn{2}{|c|}{ Test } & \multicolumn{2}{|c|}{ Learning } & \multicolumn{2}{|c|}{ Test } \\
\hline & RMSE & $\mathrm{R}^{2}$ & RMSE & $\mathrm{R}^{2}$ & RMSE & $\mathrm{R}^{2}$ & RMSE & $\mathrm{R}^{2}$ \\
\hline WA-SVR & 0.0632 & 0.8249 & 0.1550 & 0.865 & 0.1295 & 0.895 & 0.1203 & 0.897 \\
\hline WA-ANN-MLP & 0.0445 & 0.8738 & 0.1167 & 0.833 & 0.1017 & 0.853 & 0.1137 & 0.865 \\
\hline WA-ANFIS & 0.0301 & 0.9398 & 0.0851 & 0.985 & 0.0798 & 0.941 & 0.0814 & 0.911 \\
\hline \multicolumn{9}{|c|}{ 2-month } \\
\hline Station & \multicolumn{4}{|c|}{ Fez-DRH } & \multicolumn{4}{|c|}{ Ain Bittit } \\
\hline \multirow{2}{*}{ Methods } & \multicolumn{2}{|c|}{ Learning } & \multicolumn{2}{|c|}{ Test } & \multicolumn{2}{|c|}{ Learning } & \multicolumn{2}{|c|}{ Test } \\
\hline & RMSE & $\mathrm{R}^{2}$ & RMSE & $\mathrm{R}^{2}$ & RMSE & $\mathrm{R}^{2}$ & RMSE & $\mathrm{R}^{2}$ \\
\hline WA-SVR & 0.1256 & 0.8011 & 0.1441 & 0.777 & 0.1752 & 0.7895 & 0.2103 & 0.7878 \\
\hline WA-ANN-MLP & 0.1017 & 0.8113 & 0.1173 & 0.802 & 0.1436 & 0.8059 & 0.0998 & 0.7981 \\
\hline WA-ANFIS & 0.0711 & 0.8982 & 0.0832 & 0.879 & 0.0915 & 0.8839 & 0.0611 & 0.864 \\
\hline \multicolumn{9}{|c|}{ 3-month } \\
\hline Station & \multicolumn{4}{|c|}{ Fez-DRH } & \multicolumn{4}{|c|}{ Ain Bittit } \\
\hline \multirow{2}{*}{ Methods } & \multicolumn{2}{|c|}{ Learning } & \multicolumn{2}{|c|}{ Test } & \multicolumn{2}{|c|}{ Learning } & \multicolumn{2}{|c|}{ Test } \\
\hline & RMSE & $\mathrm{R}^{2}$ & RMSE & $\mathrm{R}^{2}$ & RMSE & $\mathrm{R}^{2}$ & RMSE & $\mathrm{R}^{2}$ \\
\hline WA-SVR & 0.1314 & 0.7852 & 0.2551 & 0.745 & 0.2323 & 0.7712 & 0.2002 & 0.7662 \\
\hline WA-ANN-MLP & 0.1242 & 0.7904 & 0.2423 & 0.766 & 0.2101 & 0.8024 & 0.1162 & 0.7985 \\
\hline WA-ANFIS & 0.0971 & 0.8426 & 0.2012 & 0.793 & 0.1152 & 0.8627 & 0.0812 & 0.8121 \\
\hline
\end{tabular}

The observed and predicted time series of SPI-3 and SPI-12 for 1 month in advance of the WAANFIS and ANFIS models, as well as the scatter plots of the test period (2005 -2014) at Fez-DRH, are illustrated in Figs. 4 and 5.

It is clear from Figs. 2 and 3 that WA-ANFIS outperforms ANFIS. The improvement of the R2 values provided by WA-ANFIS, for 1 month in International Journal of Intelligent Engineering and Systems, Vol.11, No.1, 2018 advance, is very relevant. From R2 $=0.782$ for ANFIS to R2 $=0.925$ for the WA-ANFIS model for the SPI-3 series and from R2 $=0.782$ for ANFIS to $\mathrm{R} 2=0.925$ for the WA-ANFIS model for the SPI12 series. This increase in R2 values by the WAANFIS model may be related to its configuration. The main idea behind the method is based on the 


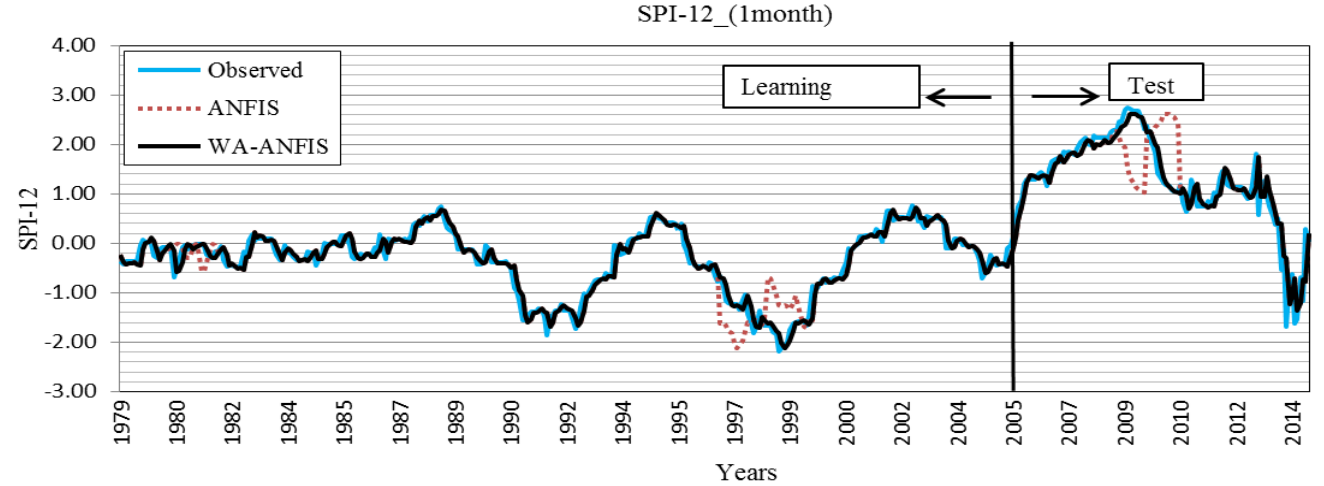

(a)

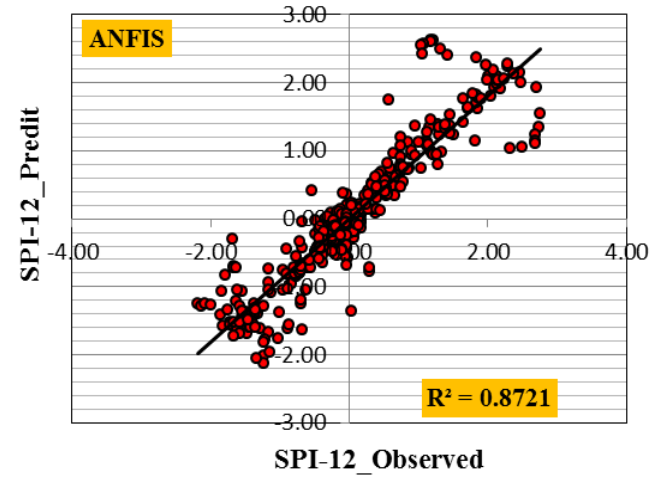

(b)

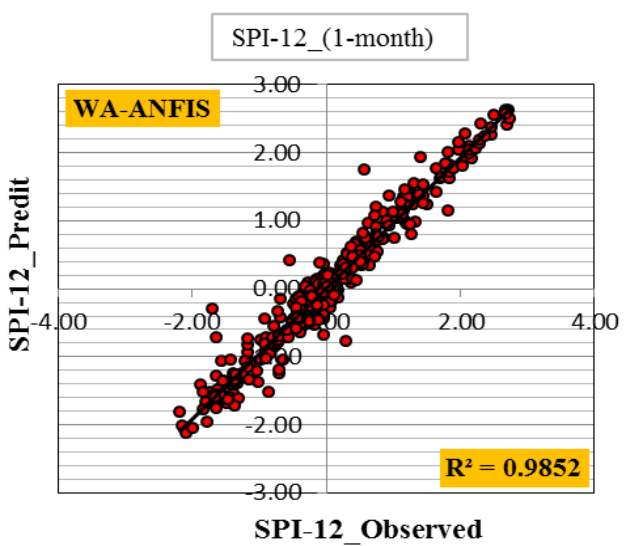

(c)

Figure.2 SPI-12 prediction results for the best WA-ANFIS model at Fez-DRH station for 1 month in advance: (a) SPI-12, (b) ANFIS, and (c) WA-ANFIS

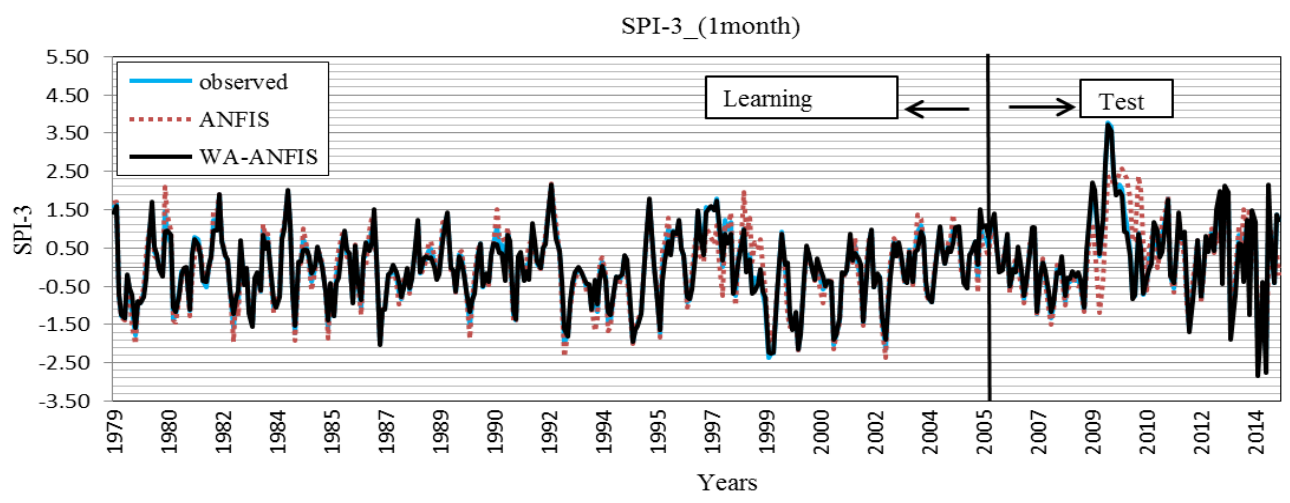

(a)

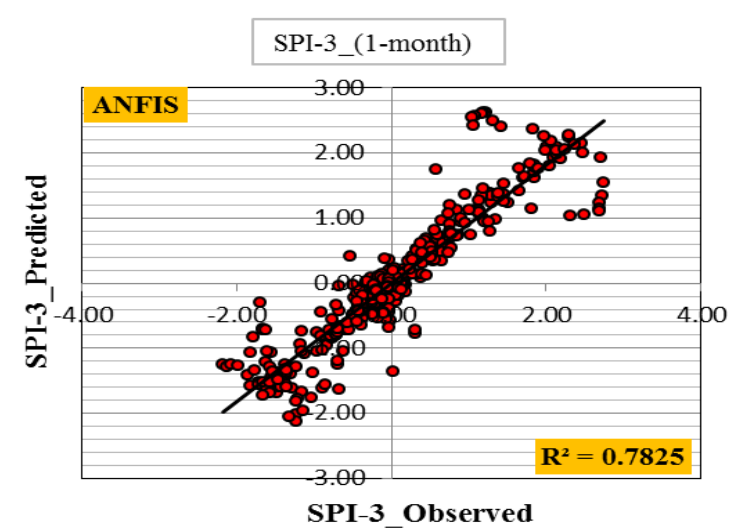

(b)

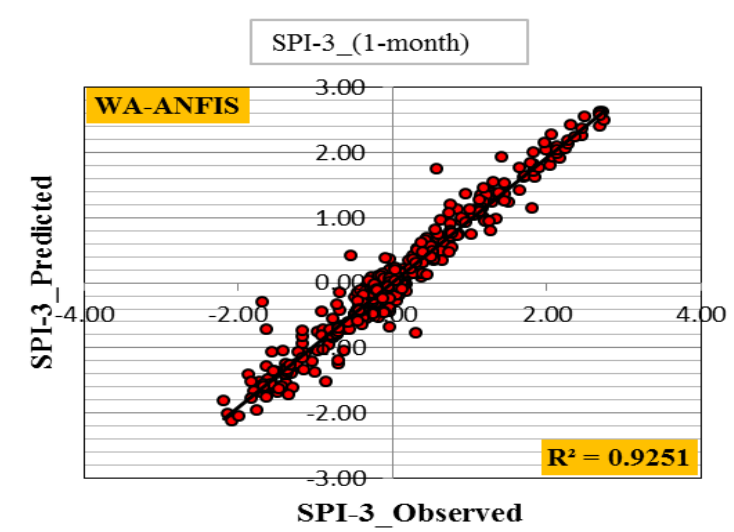

(c)

Figure.3 SPI-3 forecast results for the best WA-ANFIS model at the Fez-DRH station for 1 month ahead: (a) SPI-3, (b) ANFIS, and (c) WA-ANFIS 
wavelet band. Because WA-ANFIS uses information to different spectral bands separately, it can capture and model the behavior of data easily compared to the simple ANFIS model.

\section{Discussion}

In this study, the accuracy of the results of the ANFIS, SVM, ANN-ANN-MLP multivariate time series and the prediction of meteorological drought was examined. The results of this study indicate that the ANFIS model outperforms the other models over a nine-month period in the weather forecast. The results of this research imply that all ANN-MLP, SVM and ANFIS models are useful means of forecasting meteorological drought that work on recorded data. Therefore, it can be noted that these models could be very useful in the ongoing monitoring of regional meteorological drought in order to manage limited water resources at different times. A comparison of model results within 9 months revealed that the highest accuracy for drought prediction is obtained by the ANFIS model which is followed by the SVM, ANN-MLP models. However, with the increase in meteorological parameters, model results would be more reliable and accurate. According to the results, the ANNMLP, SVM and ANFIS models show more capacity and sensitivity to drought predictions in a 3-month period. Based on the results obtained for a period of 3 months, it was concluded that droughts in the region are more frequent but less sustainable.

The discrete wavelet transform allowed most "noisy" data to be eliminated and facilitates the extraction of quasi-periodic and periodic signals in the original data time series [20]. In general, WAANFIS models had the best forecasting results in terms of R2 and RMSE for both stations. The WASVR and WA-ANN models had the second best forecast results.

Although the WA-SVR, WA-ANN and WAANFIS models were effective in predicting SPI-3, most WA-ANFIS models had more accurate predictions. Moreover, as shown in Figs. 2 and 3, WA-ANFIS predictions appear to be more effective in predicting extreme values of SPI, whether severe or heavy precipitation.

In addition, the expected results for SPI 12 are better than the expected results for SPI 3 in almost all cases. The forecasting results for SPI-3 were low in terms of the coefficient of determination, probably because of the low autocorrelation levels of the data sets versus SPI-12.
Although the WA-SVR model closely mirrors observed trends in SPI, it appears to underestimate extreme drought events in relation to ANN. The reason why WA-ANN models appear to be slightly more effective than WA-SVR models and appear to be more effective in the prediction of extreme events is probably due to the low effectiveness of SVRs over RNA models, such as their simplicity in terms of development and their reduced computational time because the wavelet analysis used for the two machine-learning techniques is the same. This observation is also supported by the fact that most ANN predictions perform better than SVR models, as shown in Tables 2 and 4.

Theoretically, the SVR models should work better than the ANN models because they respect the principle of minimizing structural risk instead of the empirical principle of risk minimization. They should, in theory, not be acceptable for local minima or maxima. However, the performance of SVR models depends greatly on the selection of the appropriate kernel and its three parameters.

The uncertainty regarding the three SVR parameters increases the number of trials required to obtain the optimal model. Due to the prolonged computation time of the SVR models, the same quantity of tests can be carried out only for the ANN models. For ANN models, even in complex systems, the relationship between input and output variables need not be fully understood.

The capacity of the WA-ANFIS coupled models compared to the ANN, ANFIS, SVR and WAveletANN models for one, one, two and three months ahead of the planned SPI was evaluated in this study. Altogether, the time series mentioned are characterized by a high behavior of non-linearity, non-stationary and seasonality. In this study, wavelet transformation approaches, ANN, ANFIS and SVR were combined to develop two hybrid models to predict SPI for different time steps. Initially, the ANN, ANFIS and SVR models were used without preprocessing. The results showed that these models may be unable to cope with the nonlinearity and seasonality behavior of the data. In the second step, the wavelet transformation was performed on the data, and the pre-processed data was used as input for the ANN and ANFIS models. This research has shown that pre-processed data can improve SPI forecasts. In addition, the results showed that the WA-ANFIS hybrid model had the best performance.

Effective models can be determined by varying the number of neurons in the hidden layer. The production of several models with variable 
architectures is not exhaustive in terms of calculation and allows a larger selection group for the optimal model.

\section{Conclusion}

In this part, the adaptive neuro-fuzzy inference system (ANFIS) has been proposed as an alternative tool for predicting meteorological drought to traditional methods such as artificial neural networks (ANN) and regression vector systems (SVR). Commonly used. Approaches to the wavelet transform, ANN-MLP and ANFIS have been combined to develop two hybrid models to predict SPI for different time scales. This study explored the prediction of short-term (SPI-3) and long-term (SPI12) drought conditions using 6 models such as SVR, ANN-MLP, ANFIS, WA-SVR, WA- MLP and WAANFIS in the Saïss Plain. The results indicate that RNA models are more efficient than SVR models in predicting this study. The use of wavelet analysis has improved the prediction results of ANN models, in particular to predict extreme events. Indeed, using a measure for relative crest error as it has been demonstrated that the relative error of the ANN model was reduced to $88 \%$ when a WN model was used. The fact that wavelet analysis is an effective tool for revealing local discontinuities helps explain why it has been more effective in predicting extreme events in the Saïss Plain. Wavelet analysis can help reduce the noise of the original time series compared to a traditional model. The fact that the waveletbased models had the best results is probably due to the fact that wavelet decomposition easily captured the non-stationary characteristics of the data.

\section{References}

[1] U.G. Bacanli, M. Firat, and F. Dikbas, "Adaptive neuro-fuzzy inference system for drought forecasting", Stoch Environ Res Risk Assess, Vol. 23, No. 8, pp. 1143-1154, 2008.

[2] M. Keskin, O. Terzi, E. Taylan, and D. Kucukyaman, "Meteorological drought analysis using data- driven models for the lakes district, Turkey", Hydrol Sci, Vol. 56, No. 6, pp. 11141124, 2009.

[3] A. Jalalkamali, H. Sedghi, and M. Manshouri, "Monthly groundwater level prediction using ANN and neuro-fuzzy models: a case study on Kerman plain, Iran", J Hydro-Inform, Vol. 13, No. 4, pp. 867-876, 2011.

[4] B. Shirmohammadi, H.R. Moradi, and V. Moosavi, "Forecasting of meteorological drought using wavelet-ANFIS hybrid model for different time steps (case study: southeastern part of east Azerbaijan province, Iran)", Nat Hazards, Vol. 69, No. 1, pp. 389-402, 2013.

[5] M.R. Zadeh and H. Tabari, "MLP-based drought forecasting in different climatic regions", Theor Appl Climatol, Vol. 09, No. 1, pp. 407-414, 2012.

[6] C.A. Santos, B.S. Morais, and G.B.L. Silva, "Drought forecast using an artificial neural network for three hydrological zones in SanFrancisco River basin, Brazil", In: Proc. of symposium Hs.2 at the Joint IAHS \& IAH convention, Hyderabad, India, Vol. 1, No. 1, pp 302-312, 2009.

[7] A.F. Marj, and A.M.J. Meijerink, "Agricultural drought forecasting using satellite images, climate indices and artificial neural network", Int J Remote Sens, Vol. 32, No. 24, pp. $9707-$ 9719, 2011.

[8] A.R.N. Shahbazi, B. Zahraie, H. Sedghi, M. Manshouri, and M. Nasseri, "Seasonal meteorological drought prediction using support vector machine", World Appl Sci J, Vol. 13, No. 6, pp. 1387-1397, 2011.

[9] M.S. Khan and P. Coulibaly, "Application of support vector machine in lake water level prediction", J Hydrol Eng, Vol. 11, No. 3, pp. 199-205, 2006.

[10] S. Haykin, Neural networks, a comprehensive foundation, $2^{\text {nd }}$ edn. Prentice-Hall, Englewood Cliffs, pp 135-155, 1999.

[11] H.R. Maier and G.C. Dandy, "The effect of internal parameters and geometry on the performance of backpropagation neural networks: an empirical study", Environ Model Softw, Vol.13, pp. 193-209, 1998.

[12] V. Vapnik, The nature of statistical learning theory, Springer, New York, 1995.

[13] M. Cimen, "Estimation of daily suspended sediments using support vector machines", Hydrol. Sci. J., Vol. 53, No. 3, pp. 656-666, 2008.

[14] O. Kisi, "Daily river flow forecasting using artificial neural networks and auto regression model", Turk J Eng Environ Sci, Vol. 29, pp. 9-20, 2003.

[15] F. Parrella, Online Support Vector Regression Master Thesis University of Genoa, 2007.

[16] L. Wang and J.M. MENDEL, "Backpropagation fuzzy system as nonlinear dynamic system identifiers", In: Proc. of the IEEE Int. Conf. on Fuzzy Systems, San Diego, CA, ÉtatsUnis, pp. 1409-1416, 1992.

[17] J.S.R. Jang, "ANFIS: adaptive network based fuzzy interface system", In: Proc IEEE Trans Syst Man Cybern, Vol. 23, pp. 665-685, 1993. 
[18] T. Rajaee, "Wavelet and ANN combination model for prediction of daily suspended sediment load in rivers", Sci Total Environ, Vol. 409, pp. 2917-2928, 2011.

[19] A.E. Ibrahimi and A. Baali, "Application of Neural Modeling and the SPI Index for the Prediction of Weather Drought in the Saïs Plain (Northern Morocco)", International Journal of Intelligent Engineering and Systems, Vol.10, No.5, pp. 1-10, 2017.

[20] A.E. Ibrahimi and A. Baali, "Comparative Study of the Three Models (ANN-PMC), (DWT-ANN-PMC) and (MLR) for Prediction of the Groundwater Level of the Surface Water Table in the Saïss Plain (North of Morocco)", International Journal of Intelligent Engineering and Systems, Vol.10, No.5, pp. 220-230, 2017. 